International Journal of Quantum Information

(C) World Scientific Publishing Company

\title{
THE GAUGE SYMMETRY OF THE THIRD KIND AND QUANTUM MECHANICS AS AN INFRARED LIMIT
}

\author{
HANS-THOMAS ELZE \\ Dipartimento di Fisica, Università di Pisa, \\ Largo Pontecorvo 3, I-56127 Pisa, Italia \\ elze@df.unipi.it
}

Received August 31, 2018

\begin{abstract}
We introduce functional degrees of freedom by a new gauge principle related to the phase of the wave functional. Thus, quantum mechanical systems are dissipatively embedded into a nonlinear classical dynamical structure. There is a necessary fundamental length, besides an entropy/area parameter, and standard couplings. For states that are sufficiently spread over configuration space, quantum field theory is recovered.
\end{abstract}

Keywords: Gauge symmetry; nonlinear Schrödinger equation; foundations of quantum mechanics.

\section{Introduction}

We present a new $\mathcal{U}(1)$ gauge symmetry "of the third kind", related to local gauge transformations in the space of field configurations underlying quantum field theory (QFT). While results are summarized here, more details may be found in Ref. 1.

Point of departure is the observation that the reduction of the potentiality described by the wave function(al) $\Psi$ to the actuality of the outcome of a measurement process has been left outside of quantum theory (in its standard form):

"..., it is an incomplete representation of real things, although it is the only one which can be built out of the fundamental concepts of force and material points (quantum corrections to classical mechanics). The incompleteness of the representation leads necessarily to the statistical nature (incompleteness) of the laws." 2

In other words, aspects of the theory that concern the concept of information have hitherto been left separate from the concepts of force and material points, the "real things" Einstein refers to. Correspondingly, this is reflected in the remarkable derivation of the kinematical setting of quantum theory from three information theoretical constraints, as discussed, for example, in the recent review by Bub: ${ }^{3}$ the "real things" do not play a role in it.

The cut between "real things" and "information about real things" appears to be due to historical contingencies, not unlike the cut between dynamical theories, describing the effects of gravity in particular, and geometrical theories of space and time, until their fusion in general relativity. 
In this note, we propose to reconsider the role of the wave functional. We assume that its phase is not only subject to global gauge transformations ("of the first kind") and local ones ("of the second kind"), related to the common field variables of QFT: There are also functional gauge transformations which are local in the space of field configurations and which attribute a physical 'charge' to $\Psi$.

We recall that local U(1) gauge invariance of the Schrödinger equation in quantum mechanics ("first quantization") is related to the electromagnetic interaction via the classical minimal coupling prescription. Two important aspects should be noted: ${ }^{1,4}$ (A) The quantum mechanical model of a charged particle interacting with the electromagnetic field descends from Maxwell's theory, via minimally coupled classical particle Hamiltonian, through its quantization, finally to the gauge invariant Schrödinger equation. Thus, there is a classical regime where the quantum theory is anchored (Copenhagen interpretation). - (B) The Schrödinger equation can be also interpreted as nonrelativistic limit of the Klein-Gordon equation. However, the latter is not acceptable as a quantum mechanical single-particle wave equation, since there are negative energy states and a 'probability' density which is not positive definite. Instead, with minimal coupling, the Klein-Gordon equation describes interacting classical (!) scalar and electromagnetic fields.

An analogous dynamical scheme exists for the functional Schrödinger equation ("second quantization"), as we shall see. It predicts the universal coupling of all fields that are variables in this equation and introduces a fundamental length.

Guided by our new gauge principle, we couple a dynamical functional $\mathcal{A}$ to the wave functional $\Psi$, generalizing the action of Dirac's variational principle. Here, the would-be-quantum sector described by $\Psi$ forms a dissipative subsystem of the enlarged structure, effectively extending it nonlinearly and nonlocally in the space of field configurations. - Nonlinear extensions of nonrelativistic quantum mechanics have been studied earlier by Bialynicki-Birula and Mycielski ${ }^{5}$ and by Weinberg ${ }^{6}$. Works of Kibble ${ }^{7}$ and of Kibble and Randjbar-Daemi ${ }^{8}$ present nonlinear extensions of scalar QFT models where parameters of the models are quantum state dependent. However, this kind of nonlinearity is not compatible with our gauge principle, which dictates a different form of nonlinearity (in $\Psi$ ) of the wave functional equation.

As we shall see, quantum theory is embedded here into a new framework, where the potentiality represented by $\Psi$ bears the character of a charge. It causes correlations of the underlying fields beyond what is encoded in their usual Lagrangians, with QFT describing an infrared limit. We speculate that a model of this kind might point in the right direction towards unifying the concepts of "real things" and "information about real things".

In distinction to other proposals that have quantum mechanics emerge from underlying dynamics $9,10,11,12,13,14,15,16,17$, our approach does not depend on a particular field theory, such as the Standard Model, or otherwise special dynamics. We share, however, the tentative conclusion that quantum theory can and should be reconstructed as an effective theory describing large-scale behavior of fundamentally deterministic degrees of freedom. Quantum states are no longer the primary degrees 
of freedom. Bell's theorem and the predicament of local hidden variable theories are circumvented, since the implied nonlocality operates at the pre-quantum level.

\section{The Gauge Invariant Functional Wave Equation}

The functional Schrödinger picture of QFT is convenient for our argument. References to related work are given in Ref. 1. - We consider a generic scalar field theory, for simplicity, and refer to Ref. 1 concerning systems with internal gauge symmetries. The functional Schrödinger equation is:

$$
i \partial_{t} \Psi[\varphi ; t]=H[\hat{\pi}, \varphi] \Psi[\varphi ; t] \equiv \int \mathrm{d}^{3} x\left\{-\frac{1}{2} \frac{\delta^{2}}{\delta \varphi^{2}}+\frac{1}{2}(\nabla \varphi)^{2}+V(\varphi)\right\} \Psi[\varphi ; t],
$$

with the Hamiltonian $H$ corresponding to the classical Hamiltonian density; mass and selfinteraction terms are included in $V(\varphi)$. Here, the classical canonical momentum conjugate to the field (coordinate) $\varphi$ is substituted by:

$$
\pi(\vec{x}) \longrightarrow \hat{\pi}(\vec{x}) \equiv \frac{1}{i} \frac{\delta}{\delta \varphi(\vec{x})},
$$

which implements the field quantization (coordinate representation).

In analogy to gauge transformations in the first quantized Schrödinger picture, we now define $\mathcal{U}(1)$ gauge transformations of the third kind by:

$$
\Psi^{\prime}[\varphi ; t] \equiv \exp (-i \Lambda[\varphi ; t]) \Psi[\varphi ; t],
$$

where $\Lambda$ denotes a time dependent real functional. Such gauge transformations are local in the space of field configurations. They differ from the usual gauge transformations in QFT. - In fact, the wave equation (1) becomes invariant under transformation (3), if new covariant derivatives are introduced:

$$
\begin{aligned}
\partial_{t} & \longrightarrow \mathcal{D}_{t} \equiv \partial_{t}+i \mathcal{A}_{t}[\varphi ; t] \\
\frac{\delta}{\delta \varphi(\vec{x})} & \longrightarrow \mathcal{D}_{\varphi(\vec{x})} \equiv \frac{\delta}{\delta \varphi(\vec{x})}+i \mathcal{A}_{\varphi}[\varphi ; t, \vec{x}]
\end{aligned}
$$

The real functional $\mathcal{A}$ is analogous to the usual vector potential. Generally, $\mathcal{A}$ depends on $t$; it is a functional of $\varphi$ in Eq. (4), while it is a functional field in Eq. (5). Distinguishing these components of $\mathcal{A}$ by subscripts, they transform according to:

$$
\begin{aligned}
\mathcal{A}_{t}^{\prime}[\varphi ; t] & \equiv \mathcal{A}_{t}[\varphi ; t]+\partial_{t} \Lambda[\varphi ; t] \\
\mathcal{A}_{\varphi}^{\prime}[\varphi ; t, \vec{x}] & \equiv \mathcal{A}_{\varphi}[\varphi ; t, \vec{x}]+\frac{\delta}{\delta \varphi(\vec{x})} \Lambda[\varphi ; t] .
\end{aligned}
$$

Then, we may also define an invariant 'field strength':

$$
\mathcal{F}_{t \varphi}[\varphi ; t, \vec{x}] \equiv \partial_{t} \mathcal{A}_{\varphi}[\varphi ; t, \vec{x}]-\frac{\delta}{\delta \varphi(\vec{x})} \mathcal{A}_{t}[\varphi ; t]
$$

i.e., $\mathcal{F}_{t \varphi}=\left[\mathcal{D}_{t}, \mathcal{D}_{\varphi}\right] / i$.

In order to give further meaning to the coupling between $\Psi$ and $\mathcal{A}$, we have to postulate a consistent dynamics for the latter. All elementary fields supposedly are 
present as the coordinates on which the wave functional depends - presently just a scalar field, besides time. We assume the following $\mathcal{U}(1)$ invariant action:

$$
\Gamma \equiv \int \mathrm{d} t \mathrm{D} \varphi\left\{\Psi^{*}\left(\mathcal{N}(\rho) \stackrel{\leftrightarrow}{i \mathcal{D}} t-H\left[\frac{1}{i} \mathcal{D}_{\varphi}, \varphi\right]\right) \Psi-\frac{l^{2}}{2} \int \mathrm{d}^{3} x\left(\mathcal{F}_{t \varphi}\right)^{2}\right\}
$$

where $\Psi^{*} \mathcal{N} \stackrel{\stackrel{\leftrightarrow}{D}_{t}}{t} \Psi \equiv \frac{1}{2} \mathcal{N}\left\{\Psi^{*} i \mathcal{D}_{t} \Psi+\left(i \mathcal{D}_{t} \Psi\right)^{*} \Psi\right\}$, and with a dimensionless real function $\mathcal{N}$ depending on the density:

$$
\rho[\varphi ; t] \equiv \Psi^{*}[\varphi ; t] \Psi[\varphi ; t] .
$$

We shall see shortly that $\mathcal{N}$ incorporates a necessary nonlinearity. The fundamental parameter $l$ has dimension $[l]=[$ length $]$, for dimensionless measure $\mathrm{D} \varphi$ and $\Psi$.

Our action generalizes the action for a Schrödinger wave functional which has been employed for applications of Dirac's variational principle to QFT before. ${ }^{1,8}$ It depends on $\Psi, \Psi^{*}, \mathcal{A}_{t}$, and $\mathcal{A}_{\varphi}$ separately. - Varying $\Gamma$ with respect to $\Psi^{*}$ (and $\Psi$ ) yields the gauge invariant $\Psi$-functional equation of motion (and its adjoint):

$$
(\rho \mathcal{N}(\rho))^{\prime} i \mathcal{D}_{t} \Psi[\varphi ; t]=H\left[\frac{1}{i} \mathcal{D}_{\varphi}, \varphi\right] \Psi[\varphi ; t],
$$

replacing Eq. (1); here $f^{\prime}(\rho) \equiv \mathrm{d} f(\rho) / \mathrm{d} \rho$. Varying with respect to $\mathcal{A}_{\varphi}$, we obtain:

$$
\partial_{t} \mathcal{F}_{t \varphi}[\varphi ; t, \vec{x}]=\frac{1}{2 i l^{2}}\left(\Psi^{*}[\varphi ; t] \mathcal{D}_{\varphi(\vec{x})} \Psi[\varphi ; t]-\Psi[\varphi ; t]\left(\mathcal{D}_{\varphi(\vec{x})} \Psi[\varphi ; t]\right)^{*}\right) .
$$

This invariant 'gauge field equation' completes the set of dynamical equations.

The nonlinear Eq. (11) preserves the normalization, i.e. $\langle\Psi \mid \Psi\rangle \equiv \int \mathrm{D} \varphi \Psi^{*} \Psi$ is conserved, while the overlap of two different states, $\left\langle\Psi_{1} \mid \Psi_{2}\right\rangle$, generally varies in time. This seems to hint at a probability interpretation, yet the continuity equation, Eq. (14) below, shows that this cannot be maintained. For $\mathcal{A} \neq 0$, also the homogeneity property does no longer hold, i.e., $\Psi$ and $z \Psi(z \in \mathbf{Z})$ present two different physical states ${ }^{5,6}$. This changes essential aspects of the measurement theory ${ }^{7}$ and indicates that here QFT is embedded in a classical framework. - Furthermore, the Hamiltonian $H$, unlike in QFT, cannot be arbitrarily shifted by a constant $\Delta E$, transforming $\Psi \rightarrow \exp (-i \Delta E t) \Psi$. - Finally, our action is invariant under spacetime translations and spatial rotations. Elsewhere, we will present $\Gamma$ in a manifestly Lorentz invariant form, given a suitable background spacetime.

Variation of $\Gamma$ with respect to $\mathcal{A}_{t}$, which is a Lagrange multiplier, yields the corresponding gauge invariant 'Gauss' law':

$$
-\int \mathrm{d}^{3} x \frac{\delta}{\delta \varphi(\vec{x})} \mathcal{F}_{t \varphi}[\varphi ; t, \vec{x}]=\frac{1}{l^{2}} \Psi^{*}[\varphi ; t] \Psi[\varphi ; t] \mathcal{N}(\rho) .
$$

This can be combined with Eq. (12) to result in the continuity equation:

$$
0=\partial_{t}(\rho \mathcal{N}(\rho))+\frac{1}{2 i} \int \mathrm{d}^{3} x \frac{\delta}{\delta \varphi(\vec{x})}\left(\Psi^{*} \mathcal{D}_{\varphi(\vec{x})} \Psi-\Psi\left(\mathcal{D}_{\varphi(\vec{x})} \Psi\right)^{*}\right),
$$


expressing local $\mathcal{U}(1)$ 'charge' conservation in the space of field configurations. The Eq. (14) implies that the total 'charge' $Q$ has to vanish at all times: ${ }^{1}$

$$
Q(t) \equiv \frac{1}{l^{2}} \int \mathrm{D} \varphi \rho \mathcal{N}(\rho)=0 .
$$

Here, the necessity of the nonlinearity becomes obvious. Without it, the vanishing total 'charge' could not be implemented.

Besides necessarily multiplying the invariant term $\Psi^{*} i \mathcal{D}_{t} \Psi$ in $\Gamma$, the nonlinearity is not yet determined. A particular choice related to an entropy functional has been studied in Ref. 1. In this case, one finds that the 'charge' density $\rho \mathcal{N}(\rho)$ is the deviation of entropy density per unit area from a reference density $\rho S / l^{2}$. Thus, also the entropy/area $S / l^{2}$ is a parameter, besides the fundamental length $l$. (Entropy per area is an essential parameter in apparently unrelated work of Padmanabhan ${ }^{18}$, suggesting that gravity is intrinsically holographic and quantum mechanical.) In any case, the timescale of the $\Psi$-functional evolution shrinks or expands in different regions of configuration space, depending on the factor $(\rho \mathcal{N}(\rho))^{\prime}$ in Eq. (11).

Two remarks are in order here: (A) The Eqs.(11)-(13) obey a weak superposition principle ${ }^{5}$ : The sum of two solutions, $\Psi_{1,2}$, that do not overlap, presents also a solution, provided that $\mathcal{A}=\mathcal{A}_{1}+\mathcal{A}_{2}$ is determined consistently. - (B) As we argued in Ref. 1, our nonlinear extension of QFT is local in the usual sense (microcausality). ${ }^{7}$ - However, suppose we integrated out the 'gauge field'. The resulting effective equation for $\Psi$ would be nonlocal in field space and in space-time.

\section{Stationary States, Separability and QFT Limit}

We study the separation of the time dependence in Eqs. (11)-(13) with the Ansatz $\Psi[\varphi ; t] \equiv \exp (-i \omega t) \Psi_{\omega}[\varphi], \omega \in \mathbf{R}$, and consistently assuming time independent $\mathcal{A}$-functionals. Thus, the Eq. (11) yields:

$$
\left(\rho_{\omega} \mathcal{N}\left(\rho_{\omega}\right)\right)^{\prime}\left(\omega-\mathcal{A}_{t}[\varphi]\right) \Psi_{\omega}[\varphi]=H\left[\frac{1}{i} \mathcal{D}_{\varphi}, \varphi\right] \Psi_{\omega}[\varphi],
$$

with $\mathcal{D}_{\varphi}=\frac{\delta}{\delta \varphi}+i \mathcal{A}_{\varphi}$ and $\rho_{\omega} \equiv \Psi_{\omega}^{*}[\varphi] \Psi_{\omega}[\varphi]$. From Eq. (12) follows:

$$
\frac{1}{2 i}\left(\Psi_{\omega}^{*}[\varphi] \mathcal{D}_{\varphi(\vec{x})} \Psi_{\omega}[\varphi]-\Psi_{\omega}[\varphi]\left(\mathcal{D}_{\varphi(\vec{x})} \Psi_{\omega}[\varphi]\right)^{*}\right)=0,
$$

which expresses the vanishing of the 'current' in the stationary situation. - Applying a time independent gauge transformation, cf. Eqs. (3), (7), the stationary wave functional can be made real. The Eq. (17) then implies $\mathcal{A}_{\varphi}=0$; consequently, $\mathcal{D}_{\varphi} \rightarrow \frac{\delta}{\delta \varphi}$ everywhere. Finally, 'Gauss' law', Eq. (13), determines $\mathcal{A}_{t}$ :

$$
\int \mathrm{d}^{3} x \frac{\delta^{2}}{\delta \varphi(\vec{x})^{2}} \mathcal{A}_{t}[\varphi]=\frac{1}{l^{2}} \rho_{\omega} \mathcal{N}\left(\rho_{\omega}\right)
$$

which has to be solved selfconsistently together with Eq. (16). - Separation of the time dependence thus leads to two coupled equations. One may guess an appropriate time independent $\Psi_{\omega}$-functional. Having an action at hand, Eq. (9), the parameters 
of such an Ansatz can be optimized by the variational principle, in analogy to Hartree approximation and semiclassical limit of QFT. Furthermore, the Eq. (18) can be solved formally by functional Fourier transformation, eliminating $\mathcal{A}_{t}$ at the expense of introducing the nonlocality mentioned before.

Turning to separability, this is an important property of linear quantum theory. It allows to combine subsystems which do not interact with each other, without creating unphysical correlations ${ }^{5,6}$. This should be preserved to the extent that is verified experimentally. - We have argued that linear QFT arises in the infrared (IR) limit, and consequently separability: ${ }^{1}$ Assume that the system is in a diffuse state, characterized by a density $\rho_{\omega}$ that is widely spread over the space of configurations of $\varphi$. For such a high entropy state, the local energy density and the 'charge density' on the right-hand side of Eq. (18) must be small. For our particular choice of nonlinearity, then, the stationary functional Schrödinger equation results, $\omega \Psi_{\omega}=H \Psi_{\omega}$, and with it the known structures of QFT. - Consequences of small violations of this linear equation, related to terms involving $\rho_{\omega}$ or $\mathcal{A}_{t}$, should be explored. Nonlinear effects become important in our framework only for states with a small uncertainty in configuration space, such that the IR limit does not apply.

Finally, we remark that two stationary solutions, $\Psi_{\omega_{1,2}}$, of the present eigenvalue problem, in general, obey a generalized orthogonality relation that reduces to the usual one of QFT in the IR limit. ${ }^{1}$

\section{Conclusions}

New gauge transformations "of the third kind" attribute a $\mathcal{U}(1)$ 'charge' to the wave functional, which leads to an embedding of quantum field theory in a larger nonlinear structure. - It differs from earlier proposals of nonlinear generalizations of quantum mechanics or QFT ${ }^{5,6,7,8}$. We tentatively interpret it as a classical one, since differently charged components of the wave functional $\Psi$, besides being governed by the usual interactions of an underlying field theory model (such as gauge theories, see Ref. 1), are coupled through a new connection functional $\mathcal{A}$. When effects of the latter are negligible, QFT is recovered.

A number of interesting problems need further study, before this proposal can stand on its own. - A theory of the observables and the measurement process needs to be worked out. It is promising that the energy-momentum tensor following from our action, Eq. (9), similarly in the presence of internal gauge symmetries, is the one of the respective QFT plus contributions due to the coupling between $\Psi$ and $\mathcal{A}$. When the latter is small, the usual observables may be useful, while the coupling might be important for the reduction or collapse of the wave functional. A solution in the case of an underlying free field theory should be possible, based on the variational principle, for example. This will be helpful to better understand effects of the new coupling. - Reparametrization invariant models are an important target. As compared to a Wheeler-DeWitt type equation, the presence of additional nonlinear terms in what replaces this equation may actually be useful. 


\section{Acknowledgments}

I wish to thank G. 't Hooft for discussions, particularly concerning the phase of the wave function. ${ }^{17} \mathrm{I}$ am grateful to $\mathrm{M}$. Genovese for the kind invitation to the Torino workshop "Advances in Foundations of Quantum Mechanics and Quantum Information with Atoms and Photons".

\section{References}

1. H.-T. Elze, Gauge symmetry of the third kind and quantum mechanics as an infrared phenomenon, quant-ph/0604142, in: "A Sense of Beauty in Physics", Festschrift for A. Di Giacomo, ed. by M. D'Elia and E. Meggiolaro (Pisa University Press, Pisa, 2006), to appear.

2. A.Einstein, Physics and Reality, The Journal of the Franklin Institute, 221, No. 3 (March 1936); reprinted in: A. Einstein, "Ideas and Opinions" (Souvenir Press, London, 1973), p. 315.

3. J. Bub, Quantum information and computation, quant-ph/0512125 .

4. D. S. Saxon, "Elementary Quantum Mechanics" (McGraw-Hill, New York, 1976).

5. I. Bialynicki-Birula and J. Mycielski, Ann. Phys. (N.Y.) 100 (1976) 62.

6. S. Weinberg, Phys. Rev. Lett. 62 (1989) 485; Ann. Phys. (N. Y.) 194 (1989) 336.

7. T. W. B. Kibble, Commun. Math. Phys. 64 (1978) 73.

8. T. W. B. Kibble and S. Randjbar-Daemi, J. Phys. A13 (1980) 141.

9. G. 't Hooft, J. Stat. Phys. 53 (1988) 323; Nucl. Phys. B342 (1990) 471; G.' 't Hooft, K. Isler and S. Kalitzin, Nucl. Phys. B386 (1992) 495.

10. G. 't Hooft, Found. Phys. Lett. 10 (1997) 105.

11. G.' 't Hooft, Quantum Mechanics and Determinism, presented at PASCOS 2001, Eighth International Symposium on Particles, Strings and Cosmology (2001), hepth/0105105 .

12. M. Blasone, P. Jizba and G. Vitiello, Phys. Lett. A287 (2001) 205; M. Blasone et al., Phys. Lett. A310 (2003) 393; H.-T. Elze and O.Schipper, Phys. Rev. D66 (2002) 044020; H.-T. Elze, Phys. Lett. A310 (2003) 110, Physica A344 (2004) 478; quantph/0306096 ; C. Wetterich, Quantum Correlations in Classical Statistics, in: ${ }^{13}$, p. 180; quant-ph/0212031; M. Blasone, P. Jizba and H. Kleinert, Braz. J. Phys. 35 (2005) 497; Phys. Rev. A71 (2005) 052507.

13. "Decoherence and Entropy in Complex Systems", ed. H.-T. Elze, Lecture Notes in Physics, 633 (Springer-Verlag, Berlin, 2004).

14. H.-T.Elze, Quantum fields, cosmological constant and symmetry doubling, hepth/0510267; Braz. J. Phys. 35 (2005) 343; A quantum field theory as emergent description of constrained supersymmetric classical dynamics, Proceedings 8th Int. Conf. "Path Integrals. From Quantum Information to Cosmology", Prague, June 6-10, 2005, to appear, hep-th/0508095; Phys. Lett. A335 (2005) 258.

15. F. Markopoulou and L. Smolin, Phys. Rev. D70 (2004) 124029.

16. S.L. Adler, "Quantum Mechanics as an Emergent Phenomenon" (Cambridge U. Press, Cambridge, 2005).

17. G. 't Hooft, The Mathematical Basis For Deterministic Quantum Mechanics, quant$\mathrm{ph} / 0604008$.

18. T. Padmanabhan, Mod. Phys. Lett. A 17, 1147 (2002). 